\title{
A priori-defined diet quality indices, biomarkers and risk for type 2 diabetes in five ethnic groups: the Multiethnic Cohort
}

\author{
Simone Jacobs ${ }^{1,2}$, Carol J. Boushey ${ }^{1}$, Adrian A. Franke ${ }^{1}$, Yurii B. Shvetsov ${ }^{1}$, Kristine R. Monroe ${ }^{3}$, \\ Christopher A. Haiman ${ }^{3}$, Laurence N. Kolonel ${ }^{1}$, Loic Le Marchand ${ }^{1}$ and Gertraud Maskarinec ${ }^{1 *}$ \\ ${ }^{1}$ Epidemiology Program, University of Hawaii Cancer Center, Honolulu, HI 96813, USA \\ ${ }^{2}$ Epidemiology and Biostatistics Unit, Institute of Public Health, Heidelberg University, 69120 Heidelberg, Germany \\ ${ }^{3}$ Department of Preventive Medicine, Keck School of Medicine, Norris Comprehensive Cancer Center, University of Southern \\ California, Los Angeles, CA, 90032, USA
}

(Submitted 19 January 2017 - Final revision received 21 May 2017 - Accepted 7June 2017)

\section{Abstract}

Dietary indices have been related to risk for type 2 diabetes (T2D) predominantly in white populations. The present study evaluated this association in the ethnically diverse Multiethnic Cohort and examined four diet quality indices in relation to T2D risk, homoeostatic model assessment-estimated insulin resistance (HOMA-IR) and biomarkers of dyslipidaemia, inflammation and adipokines. The T2D analysis included 166550 white, African American, Native Hawaiian, Japanese American and Latino participants (9200 incident T2D cases). Dietary intake was assessed at baseline using a quantitative FFQ and T2D status was based on three self-reports and confirmed by administrative data. Biomarkers were assessed about 10 years later in a biomarker subcohort ( $n$ 10 060). Sex- and ethnicity-specific hazard ratios were calculated for the Healthy Eating Index-2010 (HEI-2010), the alternative HEI-2010 (AHEI-2010), the alternate Mediterranean diet score (aMED) and the Dietary Approaches to Stop Hypertension (DASH). Multivariable-adjusted means of biomarkers were compared across dietary index tertiles in the biomarker subcohort. The AHEI-2010, aMED (in men only) and DASH scores were related to a 10-20\% lower T2D risk, with the strongest associations in whites and the direction of the relationships mostly consistent across ethnic groups. Higher scores on the four indices were related to lower HOMA-IR, TAG and C-reactive protein concentrations, not related to leptin, and the DASH score was directly associated with adiponectin. The AHEI-2010 and DASH were directly related to HDL-cholesterol in women. Potential underlying biological mechanisms linking diet quality and T2D risk are an improved lipid profile and reduced systemic inflammation and, with regards to DASH alone, an improved adiponectin profile.

Key words: Adipokines: A priori dietary indices: C-reactive protein: Dyslipidaemia: Ethnicity: Prospective cohorts: Type 2 diabetes

Consuming a high-quality diet has been inversely associated with risk for type 2 diabetes (T2D) ${ }^{(1,2)}$. One way of assessing dietary quality is by the use of a priori dietary indices that score adherence to dietary recommendations based on scientific evidence for chronic diseases ${ }^{(3)}$. Inflammation markers have been investigated as potential mediators of the association between diet quality and T2D risk, based mainly on studies of the Mediterranean diet and, to a smaller extent, the Healthy Eating Index (HEI)-2010 ${ }^{(4)}$. The literature on other diet quality indices, such as the Dietary Approaches to Stop Hypertension (DASH) and the Alternative HEI (AHEI)-2010 is limited ${ }^{(4)}$. As part of the mechanism for a protective effect against T2D, a high diet quality may also modify T2D-related ${ }^{(5,6)}$ metabolic parameters other than inflammation markers: for example, dyslipidaemia markers and adipokines ${ }^{(4,7)}$

Given the high rates of T2D in non-white ethnic groups in the $\mathrm{USA}^{(8)}$ and around the world ${ }^{(9)}$, ways of preventing the disease are of great interest. In a previous analysis within the Hawaii part of the Multiethnic Cohort (MEC) ${ }^{(10)}$, we showed significant inverse associations between higher DASH index scores and risk for T2D in white men and women, as well as in Japanese American women. We also found a lower T2D risk for better adherence to the AHEI-2010 and alternate Mediterranean diet score (aMED) in white participants, and no associations for the HEI-2010 score.

The current analysis applies an updated T2D definition and expands the previous report of a priori dietary indices with

Abbreviations: AHEI, Alternative Healthy Eating Index; aMED, alternate Mediterranean diet score; CRP, C-reactive protein; DASH, Dietary Approaches to Stop Hypertension; HEI, Healthy Eating Index; HOMA-IR, homoeostatic model assessment-estimated insulin resistance; MEC, Multiethnic Cohort; QFFQ, quantitative FFQ; T, tertile; T2D, type 2 diabetes.

*Corresponding author: G. Maskarinec, fax +1 808586 2982, email gertraud@cc.hawaii.edu 
incident T2D to African Americans and Latinos and to the California part of the MEC. In addition, we examine, in a subset of 10060 cohort participants with biomarker data, the association of the four dietary indices with five T2D-related ${ }^{(5,6)}$ biomarkers reflecting different metabolic pathways including adipokines with probable influence on insulin sensitivity (adiponectin) and insulin secretion (leptin) ${ }^{(11)}$, dyslipidaemia (HDL-cholesterol, TAG) and inflammation (C-reactive protein (CRP)), as well as with homoeostatic model assessmentestimated insulin resistance (HOMA-IR) whose high values reflect a pre-diabetic stage.

\section{Methods}

\section{Study population}

The MEC recruited participants from five ethnic groups (African Americans, Japanese Americans, Latinos, Native Hawaiians, whites) living in Hawaii or California (primarily in Los Angeles) during 1993-1996 ${ }^{(12)}$. More than 215000 men and women aged 45-75 years at recruitment returned a mailed self-administered survey consisting of a quantitative FFQ (QFFQ) and items on demographics, medical conditions, anthropometric measures and lifestyle factors ${ }^{(12)}$. The institutional review boards at the University of Southern California and the University of Hawaii approved the study protocol. Regular linkages with state death certificate files in Hawaii and California and the National Death index have been performed to update vital status.

\section{Dietary indices}

Using data from the validated QFFQ that included ethnicityspecific foods and a large database of local recipes ${ }^{(13,14)}$, four a priori-defined and selected dietary indices were calculated within the MEC as described in detail previously ${ }^{(15)}$. The HEI-2010 gives higher scores for better adherence to federal dietary guidelines ${ }^{(16)}$ and updates the components used in the development of HEI-2005 ${ }^{(17)}$ with modifications to components measuring the intake of seafood and plant protein, vegetables and beans, refined grains as well as the ratio of PUFA and MUFA:SFA ${ }^{(16)}$. All twelve components except the fatty-acid ratio were calculated as per $4184 \mathrm{~kJ}(1000 \mathrm{kcal})$, and points of 5, 10 or 20 were assigned to optimal intakes. The eleven components of the AHEI-2010 take into account foods and nutrients that have been consistently related to a lower risk for chronic diseases in the scientific literature as reviewed since the establishment of the first $\mathrm{AHEI}^{(18)}$. Red and processed meat, sugar-sweetened beverages (SSB) and $\mathrm{Na}$ are reverse-coded such that lower intakes provide the maximum points. The aMED includes nine components with MEC-specific medians as cut-off points and builds on previous versions of the Mediterranean diet ${ }^{(19,20)}$. The DASH index, as outlined by Fung et $a l^{(21)}$, includes eight components that are relevant in a diet used for hypertension management ${ }^{(21)}$. For the DASH index, scoring is based on intake quintiles created within the entire MEC data set; participants in the lowest quintile received 1 point and individuals in quintile five received 5 points. Red and processed meat, SSB and $\mathrm{Na}$ were reverse-coded.

\section{Case ascertainment}

Self-reported T2D status was obtained from three questionnaires that included the question 'Has your doctor ever told you that you had diabetes?' administered at cohort entry in 1993-1996, in a short follow-up survey in 1999-2002 and in a full questionnaire in 2003-2007. In addition, three sources of administrative data were available: Medicare claims on 114309 cohort members who were fee-for-service beneficiaries ${ }^{(22)}$, a 2007 linkage with diabetes care registries of the two major health insurance plans in Hawaii ${ }^{(23)}$ and hospital discharge diagnosis data in California ${ }^{(24)}$. MEC members who selfreported $\mathrm{T} 2 \mathrm{D}$ in any questionnaire after cohort entry and those who were confirmed as cases by one of the administrative data sources were defined as incident cases; an estimated year of T2D discovery was assigned based on the first report of a diagnosis. Although not perfect, self-reports of T2D have been found to be quite accurate (80-98\%) in several large prospective cohorts and in a Mayo patient study ${ }^{(25-28)}$. Nevertheless, 2258 participants with self-reported T2D who were not part of any administrative data were considered non-cases in our analysis to avoid misclassification and to maximise specificity of the T2D definition. When we tested different models (data not shown), the approach of using a concordant definition resulted in the best prediction models; not only was the small proportion of false positives excluded but also self-reported cases confirmed by administrative data probably represent more serious disease.

\section{Biomarker assays}

In 2001-2006, a MEC biospecimen subcohort of 68740 cohort members ( $49.7 \%$ of eligible) was established. Blood samples from controls in three different case-control studies were assessed for biomarkers ( $n$ 12578). Adiponectin and leptin concentrations from plasma were measured using ELISA kits (Cat No. DRP300; R\&D Systems, Inc.). Insulin concentration in serum was measured using an ELISA kit (Cat No. EZHI-14K; EMD Millipore). All ELISA protocols were in accordance with the manufacturer's instructions. A Cobas Mira Plus chemistry autoanalyser (Roche Diagnostics) was used to measure serum concentrations of glucose (Randox), CRP, total cholesterol, HDL-cholesterol and TAG (Pointe Scientific, Inc.) as per the manufacturer's instructions. HOMA-IR was calculated as (fasted insulin $(\mathrm{mU} / \mathrm{l}) \times$ fasted glucose $(\mathrm{mg} / \mathrm{dl})) / 405$.

\section{Statistical analysis}

Of 215831 participants with information on T2D status, 49281 participants were excluded, with some overlap: all participants who reported T2D at cohort entry ( $n$ 28153), members of other ethnicity ( $n$ 13994) and individuals with missing values for essential covariates ( $n$ 11987). The analysis of dietary indices with diabetes incidence, therefore, included 74693 men and 91857 women. For the biomarker subcohort, the same exclusion criteria were applied and, additionally, prevalent T2D cases at blood draw ( $n$ 2117) and participants with missing biomarker information ( $n$ 90) were excluded resulting in a sample size of 4661 men and 5399 women. 
All analyses were conducted using SAS version 9.4 (SAS Institute, Inc.).

Cox proportional hazards regression, using follow-up time as the underlying time metric and stratified by continuous age at cohort entry (PROC PHREG procedure in SAS), was applied to estimate hazard ratios (HR) and 95\% CI using the sex-specific tertiles (T1-T3) of the dietary index scores. The Cox proportional hazard assumption was verified by plotting Schoenfeld residuals. Follow-up time was calculated as the time between cohort entry and T2D discovery for participants with selfreports confirmed by administrative data (using date of selfreport or claim, whichever came first), date of death or last date of study (12/31/2010). The models were adjusted for known T2D risk factors, including ethnicity (whites as the reference), moderate/vigorous physical activity $(<30$ and $\geq 30 \mathrm{~min} / \mathrm{d}$ ), smoking (current, past and never smoker), years of education $(<12,12,13-15$ and $\geq 16$ years), total energy intake $(\mathrm{kJ} / \mathrm{d}(\mathrm{kcal} / \mathrm{d}))$ and BMI calculated from self-reported height and weight.

We used the log transformations for TAG, CRP, leptin, HOMA-IR and adiponectin to normalise the right-skewed distributions.

For the biomarker analysis, general linear models (PROC GLM in SAS) were applied to compare geometric means of the five biomarkers and of HOMA-IR, by sex-specific tertiles of dietary indices. The models were adjusted for the same covariates as in the Cox regression. A possible dose-response relation was assessed using trend tests with the tertile medians as continuous variables. Statistical inference was based on ANOVA type-III $F$ tests. The number of incident T2D cases within the biomarker subcohort was too small for analysis, including for a formal mediation analysis.

\section{Results}

The 74693 men and 91857 women in the analytical cohort had very similar characteristics to the full MEC cohort before exclusions (Table 1). The overall risk for T2D in men and women combining the five ethnic groups is lower by $7-20 \%$ across the four indices when extreme tertiles are compared (Fig. 1). The association with T2D is statistically significant for DASH and AHEI-2010 in men and women, and the aMED only in men, but NS for the HEI-2010 index in either sex group. The association appears strongest for the DASH, with HR of 0.81 (95\% CI $0.75,0.88)$ in men and 0.80 (95\% CI $0.73,0.86)$ in women. In ethnicity-specific analyses, the direction of the relations is mostly consistent across ethnic groups with a few exceptions (e.g. HEI-2010 and aMED in Native Hawaiian and Japanese American women). However, the associations are significant for the DASH index in white and Latino men and white and Japanese American women, for the AHEI-2010 in white women and African American men, for the aMED in Japanese American men and for the HEI-2010 in white women.

The biomarker subcohort includes 4661 men and 5399 women (Table 1), which is $6 \%$ of the cohort members included in the diet-T2D analysis. The mean time between cohort entry and blood draw was 9.5 (SD 2.2) years. By design, the ethnic distribution differs greatly: the proportion of whites and
Japanese Americans is smaller, whereas the percentage of Native Hawaiians and Latinos is greater than that in the full cohort. The biomarker subcohort also has fewer current smokers but is relatively similar in age, BMI, education and lifestyle factors to the full cohort. Compared with men, women have higher median HEI-2010, AHEI-2010 and DASH scores, $53 \%$ higher adiponectin, $50 \%$ higher CRP, 23\% higher HDLcholesterol and $>3$ times higher median leptin concentrations. In the subcohort, lower HOMA-IR values for higher diet scores across all indices are seen (Table 2); however, the associations did not reach significance for the HEI-2010 and aMED in women and for AHEI-2010 in men.

Only the DASH index (Fig. 2) shows a statistically significant trend for higher adiponectin concentrations, consistent in men (T1: $5 \cdot 2 \mu \mathrm{g} / \mathrm{ml}(95 \%$ CI $5 \cdot 1,5 \cdot 4)$, T2: $5 \cdot 5 \mu \mathrm{g} / \mathrm{ml}$ (95\% CI 5.3, 5.7), T3: $5.6 \mu \mathrm{g} / \mathrm{ml}(95 \%$ CI $5.4,5.7)$ ) and women (T1: $8.0 \mu \mathrm{g} / \mathrm{ml}$ (95\% CI 7.8, 8.2), T2: $8.3 \mu \mathrm{g} / \mathrm{ml}(95 \%$ CI 8.0, 8.5), T3: $8.4 \mu \mathrm{g} / \mathrm{ml}$ (95\% CI 8.1, 8.6)). None of the four dietary index scores was associated with leptin. HDL-cholesterol means show statistically significant positive trends in women, but not men, for increasing AHEI-2010 and DASH scores (AHEI-2010: T1: 1.29 (95\% CI 1.27, 1.31) mmol/l, T2: 1.31 (95\% CI 1.29, 1.33) mmol/l, T3: 1.32 (95\% CI 1.30, 1.34) mmol/l, DASH: T1: 1.29 (95\% CI $1 \cdot 27,1 \cdot 31) \mathrm{mmol} / \mathrm{l}, \mathrm{T} 2: 1.31$ (95\% CI $1.29,1.33) \mathrm{mmol} / \mathrm{l}, \mathrm{T} 3: 1.33$ (95\% CI $1.31,1.35) \mathrm{mmol} / \mathrm{l}$ ).

All four index scores show significant inverse trends with TAG in both men and women. For CRP, all four index scores indicate inverse trends in both sexes, and except for two of the index scores in women (HEI-2010 and AHEI-2010), all were statistically significant. The differences between extreme tertiles of CRP range between 12 and $18 \%$ in men, and between 4 and $8 \%$ in women. All biomarker means were adjusted for anthropometric and demographic covariates and total energy intake.

\section{Discussion}

In the current prospective analyses among five ethnic groups in the MEC, diet quality, as assessed by four a priori indices, was associated with T2D risk in the diabetes cohort and with lower values of HOMA-IR (a marker for insulin resistance, a prediabetic stage) measured, on average, 10 years after dietary assessment at cohort entry in the biomarker subcohort. The inverse associations with T2D were stronger for whites than for African Americans, Japanese Americans, Latinos and Native Hawaiians. As one of the first prospective analyses relating a priori dietary indices to diabetes-related markers, the findings that CRP and TAG concentrations were lower in participants with higher diet quality (as measured by all four indices), and that adiponectin concentration was higher in participants with higher dietary index scores (as measured by the DASH index in particular), suggest possible pathways by which high-quality diets may translate to lower T2D risk.

The statistically significant inverse association between all dietary index scores (except AHEI-2010) and HOMA-IR values in men and between AHEI-2010, DASH and HOMA-IR values among women adds to the scarce literature on diet quality and HOMA-IR ${ }^{(29)}$. A recent meta-analysis of dietary trials, however, 
Table 1. Characteristics of the Multiethnic Cohort (MEC) and the MEC biomarker subcohort (Mean values and standard deviations)

\begin{tabular}{|c|c|c|c|c|c|c|c|c|c|c|c|c|}
\hline \multirow[b]{3}{*}{ Characteristics } & \multicolumn{4}{|c|}{ Full cohort $(1993-1996)^{\star}$} & \multicolumn{4}{|c|}{ Diabetes cohort (1993-1996)† } & \multicolumn{4}{|c|}{ Biomarker subcohort (2001-2006)‡ } \\
\hline & \multicolumn{2}{|c|}{ Men } & \multicolumn{2}{|c|}{ Women } & \multicolumn{2}{|c|}{ Men } & \multicolumn{2}{|c|}{ Women } & \multicolumn{2}{|c|}{ Men } & \multicolumn{2}{|c|}{ Women } \\
\hline & Mean & SD & Mean & SD & Mean & SD & Mean & SD & Mean & SD & Mean & SD \\
\hline$n$ & \multicolumn{2}{|c|}{96963} & \multicolumn{2}{|c|}{118868} & \multicolumn{2}{|c|}{74693} & \multicolumn{2}{|c|}{91857} & \multicolumn{2}{|c|}{4661} & \multicolumn{2}{|c|}{5399} \\
\hline HEI-2010 score & 65.4 & 11.0 & $69 \cdot 3$ & 10.9 & $65 \cdot 3$ & $11 \cdot 1$ & 69.4 & 11.0 & 65.9 & $10 \cdot 8$ & $70 \cdot 3$ & $10 \cdot 7$ \\
\hline HEl-2010 score (range) & \multicolumn{2}{|c|}{$13 \cdot 4-100$} & \multicolumn{2}{|c|}{$20 \cdot 3-100$} & \multicolumn{2}{|c|}{$13 \cdot 4-100$} & \multicolumn{2}{|c|}{$20 \cdot 3-100$} & \multicolumn{2}{|c|}{$30 \cdot 3-95 \cdot 9$} & \multicolumn{2}{|c|}{$26 \cdot 4-96 \cdot 8$} \\
\hline AHEI-2010 score & $63 \cdot 7$ & 9.7 & 64.5 & $9 \cdot 2$ & $63 \cdot 5$ & 9.8 & 64.4 & $9 \cdot 2$ & 63.9 & 9.5 & $65 \cdot 0$ & $9 \cdot 0$ \\
\hline AHEI-2010 score (range) & $25 \cdot 1$ & -101 & $28 . C$ & -101 & $25 \cdot 1$ & -101 & 28.0 & $-99 \cdot 7$ & 31.9 & 99.4 & 30 & \\
\hline aMED score & 4.2 & 1.8 & $4 \cdot 1$ & 1.8 & $4 \cdot 1$ & 1.8 & 4.1 & 1.8 & $4 \cdot 2$ & 1.8 & $4 \cdot 2$ & 1.8 \\
\hline aMED score (range) & & -9 & & -9 & 0 & & & -9 & & & & \\
\hline DASH score & $24 \cdot 0$ & 4.4 & $24 \cdot 0$ & 4.4 & 23.9 & 4.5 & 24.0 & 4.4 & 23.9 & 4.4 & $24 \cdot 1$ & $4 \cdot 3$ \\
\hline DASH score (range) & & & & -39 & 9- & & & -39 & & & & \\
\hline Age at cohort entry (years) & $60 \cdot 3$ & $8 \cdot 8$ & $59 \cdot 7$ & 8.8 & $59 \cdot 8$ & 8.9 & $59 \cdot 3$ & $8 \cdot 8$ & $59 \cdot 0$ & 8.2 & 57.9 & $8 \cdot 2$ \\
\hline Age at blood draw (years) & & & & & & & & & $68 \cdot 3$ & $8 \cdot 1$ & $67 \cdot 6$ & $8 \cdot 3$ \\
\hline Ethnicity (\%) & & & & & & & & & & & & \\
\hline White & & 3.7 & & 2.4 & 27 & & & 6.0 & & & & \\
\hline African American & & 3.2 & & 3.7 & 13 & & & 3.4 & & & & \\
\hline Native Hawaiian & & 5 & & 3.9 & & 6 & & $7 \cdot 1$ & & & & \\
\hline Japanese American & & .8 & & 5.3 & 3( & & & 8.0 & & & & \\
\hline Latinos & & .5 & & 0 & 23 & & & 0.4 & & & & \\
\hline Others & & 3 & & 5.7 & $\Lambda$ & & $\Lambda$ & IA & & & & \\
\hline BMI $\left(\mathrm{kg} / \mathrm{m}^{2}\right)$ & $26 \cdot 6$ & $4 \cdot 2$ & 26.5 & $5 \cdot 8$ & $26 \cdot 4$ & 4.0 & $26 \cdot 1$ & 5.5 & $26 \cdot 6$ & 3.9 & $26 \cdot 4$ & $5 \cdot 2$ \\
\hline Energy intake (kJ) & 10138 & 4648 & 8293 & 3962 & 10138 & 4648 & 8293 & 3962 & 10418 & 4828 & 8523 & 4180 \\
\hline Physical activity & & & & & & & & & & & & \\
\hline$<30 \mathrm{~min} / \mathrm{d}$ & & 2 & & 3.6 & 3 & & 4 & 0.0 & & & & \\
\hline$\geq 30 \mathrm{~min} / \mathrm{d}$ & & 38 & & 5.4 & 65 & & 5 & 7.9 & & & & \\
\hline Smoking status (\%) & & & & & & & & & & & & \\
\hline Never & & .0 & & 5.7 & 3( & & & 4.9 & & & & \\
\hline Past & & .7 & & $9 \cdot 2$ & 5 & & & 9.1 & & & & \\
\hline Current & & 3 & & $4 \cdot 2$ & $1 \varepsilon$ & & & $4 \cdot 3$ & & & & \\
\hline Educational status (\%) & & & & & & & & & & & & \\
\hline$\leq 12$ years & & & & 5.8 & 4( & & & 5.0 & & & & \\
\hline $13-15$ years & & 8 & & 3.8 & $2 \varsigma$ & & & 0.0 & & & & \\
\hline$\geq 16$ years & & 3.9 & & 4.4 & 3 & & 2 & 5.1 & & & & \\
\hline Biomarkers (medians and int & rquartile & anges) & & & & & & & & & & \\
\hline Adiponectin $(\mu \mathrm{g} / \mathrm{ml})$ & & $A$ & & A & $\Lambda$ & & & JA & 5.5 & 4.9 & 8.4 & 7.4 \\
\hline Leptin $(\mu \mathrm{g} / \mathrm{l})$ & & A & & IA & 1 & & & JA & 6.5 & $8 \cdot 2$ & $22 \cdot 4$ & 24.5 \\
\hline HDL-cholesterol $(\mathrm{mmol} / \mathrm{l})$ & & A & & IA & $\mathrm{N}$ & & & JA & 1.0 & 0.44 & 1.24 & 0.54 \\
\hline TAG $(\mathrm{mmol} / \mathrm{l})$ & & A & & IA & 1 & & & JA & 1.12 & 0.87 & $1 \cdot 13$ & 0.80 \\
\hline C-reactive protein $(\mathrm{mmol} / \mathrm{l})$ & & A & & IA & $\Lambda$ & & & JA & $13 \cdot 3$ & 21.9 & $20 \cdot 0$ & 32.4 \\
\hline HOMA-IR§ & & A & & IA & 1 & & & JA & 1.3 & 1.3 & 1.4 & 1.4 \\
\hline
\end{tabular}

* Missing values in women: dietary indices, 5072; BMI, 2326; energy intake, 5071; physical activity, 3220; smoking status, 2328; educational status, 1519. Missing values in men: dietary indices, 4080; BMI, 789; energy intake, 4081; physical activity, 1667; smoking status, 1134; educational status, 1068.

† Missing values in women: physical activity, 1909; smoking status, 1542. Missing values in men: physical activity, 950; smoking status, 759

‡ Missing values in women: physical activity, 87; smoking status, 82. Missing values in men: physical activity, 45; smoking status, 42.

$\S$ Participants with missing fasting insulin and glucose values were excluded for homoeostatic model assessment-estimated insulin resistance analysis (included in analysis: men, 4419 and women, 5224).

reported no significant effect of the DASH diet on HOMA-IR compared with the control diet, although the diet lowered fasting insulin when prescribed for more than 16 weeks ${ }^{(30)}$.

Higher adherence to AHEI-2010, aMED (in men only) and DASH scores was related to a $10-20 \%$ significant lower T2D risk in the present analysis. Regarding ethnicity-specific findings, white men and women, Japanese American women and Latino men exhibited significant inverse associations between the DASH index score and T2D risk for the highest $v$. the lowest tertile. Other studies reported mixed findings: for example, the Women's Health Initiative (WHI), with participants being older at cohort entry than MEC women, observed an inverse association between the DASH index and T2D risk in white, African American, Hispanic and Asian women, although it did not reach statistical significance in the small group of Asians when comparing extreme quintiles ${ }^{(31)}$. The Insulin Resistance Atherosclerosis Study reported an inverse association between the DASH index and T2D risk only in whites and not in African Americans or Hispanics, but the latter two groups were smaller than those in our study ${ }^{(32)}$. Several reports with whites also showed null associations ${ }^{(33,34)}$.

The inverse association between the AHEI-2010 and T2D risk in white women in the present study was similar to findings in the $\mathrm{WHI}^{(31)}$ and in the Nurses' Health Study (NHS) ${ }^{(35)}$; however, contrary to our findings, significant associations in African Americans and Hispanics, though not in Asians, were observed in the WHI. The AHEI-2010 score was associated with a $20 \%$ reduction in T2D risk among white women in the present study, 


\begin{tabular}{|c|c|c|c|c|c|c|c|}
\hline & Men & & & & Womer & & \\
\hline HEI-2010 & & & HR $(95 \% \mathrm{Cl})$ & HEl-2010 & & & $\mathrm{HB}(9$ \\
\hline All & 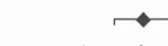 & & $0.93(0.86,1.00)$ & All & - & & $0.86,1.00)$ \\
\hline White & $\longmapsto$ & $\longrightarrow$ & $0.92(0.78,1.09)$ & White & $\longmapsto$ & & $0.82(0.69,0.98)$ \\
\hline African American & $\longmapsto$ & & $0.99(0.79,1.24)$ & African American & $\longmapsto$ & — & $1.06)$ \\
\hline Native Hawaiian & $\bullet$ & $\longrightarrow$ & $0.93(0.72,1.20)$ & Native Hawaiia & & $\bullet$ & $1 \cdot 29)$ \\
\hline Japanese American & $\longmapsto$ & $\longrightarrow$ & $0.97(0.84,1.11)$ & Japanese $\mathrm{Ar}$ & $\vdash$ & 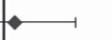 & $.18)$ \\
\hline Latino & $\longmapsto$ & $\longrightarrow$ & $0.91(0.77,1.06)$ & Latino & $\rightarrow$ & $\multimap$ & $6,1 \cdot 07)$ \\
\hline AHEI-2010 & & & & AHEI-2010 & & & \\
\hline All & $\longmapsto$ & & $0.85(0.79,0.92)$ & All & $\mapsto \bullet$ & & $.83,0.97)$ \\
\hline White & $\longmapsto$ & 十 & $0.90(0.76,1.05)$ & White & $\longmapsto$ & & 0.80 \\
\hline African American & $\longmapsto$ & & $0.75(0.59,0.96)$ & African American & $\longmapsto$ & $\mapsto$ & $0.89(0.75,1.06)$ \\
\hline Native Hawaiian & $\longmapsto$ & -1 & $0.82(0.64,1.06)$ & Native Hawaiian & $\longmapsto$ & $\longrightarrow$ & $0.88(0.71,1.10)$ \\
\hline Japanese American & $\longmapsto$ & $-H$ & $0.90(0.79,1.03)$ & Japanese American & $\longmapsto$ & $\longrightarrow$ & $33,1 \cdot 11)$ \\
\hline Latino & $\longmapsto$ & & $0.85(0.72,1.00)$ & Latino & 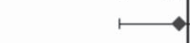 & $\longrightarrow$ & $0.98(0.83,1.15)$ \\
\hline aMED & & & & & & & \\
\hline All & $\longmapsto$ & & $0.85(0.77,0.92)$ & All & $\mapsto$ & & 0.93 \\
\hline White & $\longmapsto$ & -1 & $0.88(0.73,1.05)$ & White & $\longmapsto$ & - & 0.89 \\
\hline African American & $\leftrightarrow$ & $\longrightarrow$ & $0.85(0.64,1.11)$ & African American & $\bullet$ & 4 & $72,1.02)$ \\
\hline Native Hawaiian & $\leftrightarrow$ & $\longrightarrow$ & $0.81(0.60,1.08)$ & Native Hawaiian & & & $1.01(0 \cdot 80,1 \cdot 28)$ \\
\hline Japanese American & $\longmapsto$ & & $0.83(0.71,0.96)$ & Japanese American & $\vdash$ & $\longrightarrow$ & $1.04(0.90,1.20)$ \\
\hline Latino & $\longmapsto$ & $\longrightarrow$ & $0.91(0.76,1.09)$ & Latino & 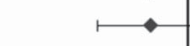 & $\longrightarrow$ & $0.90(0.77,1.06)$ \\
\hline DASH & & & & DASH & & & \\
\hline $\begin{array}{l}\text { All } \\
\text { White }\end{array}$ & $\mapsto \bullet$ & & $0.81(0.75,0.88)$ & All & $\mapsto$ & & $0.80(C$ \\
\hline African America & 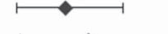 & & $0.14(0.02,0.08)$ & White & $\checkmark$ & & $0.68(0.56,0.83$ \\
\hline Native Hawaiian & $\bullet$ & + & $0.87(0.67,1.12)$ & African American & $\longmapsto \bullet$ & + & $\begin{array}{l}0.85(0.10,1.02 \\
0.88(0.68,1.13\end{array}$ \\
\hline Japanese American & $\longmapsto$ & & $0.89(0.78,1.02)$ & Japanese American & $\longmapsto$ & & $0.85(0.73,0.99$ \\
\hline Latino & $\longmapsto$ & & $0.82(0.70,0.97)$ & Latino & $\longmapsto$ & & $0.84(0.70,1.00$ \\
\hline $\begin{array}{lll}.00 & 0.20 & 0.40\end{array}$ & 0.80 & & $\begin{array}{lll}40 & 1.60 & 1.80\end{array}$ & $\begin{array}{lll}0.00 & 0.20 & 0.40\end{array}$ & 0.80 & & $1.40 \quad 1.60$ \\
\hline
\end{tabular}

Fig. 1. Association of Dietary Index Scores with type 2 diabetes risk by sex and ethnicity in the Multiethnic Cohort. Values are hazard ratios (HR) and $95 \% \mathrm{Cl}$ for tertile (T) 3 compared with T1 (reference) of the dietary index scores stratified by age and adjusted for physical activity, smoking status, education, total energy intake and BMI, separately for men ( $n$ 74693) and women $(n$ 91 857) and by ethnic group. Models for men or women combining ethnic groups were additionally adjusted for ethnicity. Dietary index score range by tertile in men: Healthy Eating Index (HEI)-2010: T1: 13.4-60.6, T2: 60.6-70.4, T3: 70.4-100; Alternative Healthy Eating Index (AHEI)-2010: T1: 25.1-59.3, T2: 59.3-67.8, T3: 67.8-101; alternate Mediterranean diet score (aMED): T1: 0-3, T2: 4-5, T3: 6-9; Dietary Approaches to Stop Hypertension (DASH): T1: 9-21, T2: 22-25, T3: 26-39; and in women: HEl-2010: T1: 20.3-65.0, T2: 65.0-74.8, T3: 74.8-100; AHEI-2010: T1: 28.0-60.5, T2: 60.5-68.5, T3: 68.5-99.7; aMED: T1: 0-3, T2: 4-4, T3: 5-9; DASH: T1: 9-21, T2: 22-26, T3: 27-39. Because of space considerations, more decimal places are not presented, but with more decimal places, the limits do not overlap.

but among white men, the null result observed in our study is in contrast to an inverse association found in another study in white men ${ }^{(36)}$. Other studies combining white men and women found no association of AHEI with T2D risk ${ }^{(33,34)}$. A previous study reported an inverse association between the aMED score and T2D for white, African American and Hispanic women ${ }^{(31)}$, which could not be replicated in the current study. Our findings disagree with an inverse association between HEI-2010 and T2D in African American women in the $\mathrm{WHI}^{(31)}$, but agree with null results for the HEI-2005 score with T2D risk in men ${ }^{(36)}$.

Different index versions used in other cohorts - for example, the HEI-2005 in the Health Professionals Follow-Up Study ${ }^{(36)}$ and the DASH eating plan in the InterAct study ${ }^{(33)}$ - as opposed to the HEI-2010 and the DASH index ${ }^{(21)}$ in the current study, may be responsible for discrepant results, as may be the diverse ethnic composition of study populations or different FFQs used in different cohorts. Also, as the DASH index relies on study-specific medians for food components, DASH scores from different studies are incomparable by definition.

Ethnic differences in the strength of associations between diet quality and T2D may be due to underlying biologic differences in the metabolism of glucose and/or insulin ${ }^{(37)}$ or to different consumption patterns of foods that may result in different nutrient composition. For example, the major source of vegetables in the MEC were tomato or vegetable soup among Latino men, starchy vegetables among African Americans and cabbage and stir fries among Japanese Americans ${ }^{(38)}$. When analysed for micronutrients, ethnic variation was seen primarily for foods contributing to vitamin $\mathrm{A}$ and $\mathrm{E}$ intake ${ }^{(38)}$. Also, the dietary indices were originally tested among persons of Caucasian and African American (for DASH) heritage and may not represent foods consumed by Japanese Americans, Native Hawaiians and Latinos. Small differences in results between the previous analysis within the Hawaii part of the MEC ${ }^{(10)}$ and the present study may be due to an updated T2D definition and a different sample size. In the previous report ${ }^{(10)}$, only information from health plans in Hawaii, but not hospital discharge data from California and Medicare data for both locations, was available.

In the search for possible underlying pathways between diet and $\mathrm{T} 2 \mathrm{D}$, we detected inverse associations of all four dietary indices with CRP and TAG, although these were not statistically 
Table 2. Association of dietary index scores with homoeostatic model assessment-estimated insulin resistance (HOMA-IR) in the Multiethnic Cohort biomarker subcohort*

\begin{tabular}{|c|c|c|c|c|c|c|}
\hline \multirow[b]{2}{*}{ Dietary Index } & \multicolumn{3}{|c|}{ Men } & \multicolumn{3}{|c|}{ Women } \\
\hline & $n$ & Mean & $95 \% \mathrm{Cl}$ & $n$ & Mean & $95 \% \mathrm{Cl}$ \\
\hline \multicolumn{7}{|l|}{ HEI-2010 } \\
\hline $\mathrm{T} 1$ & 1465 & 1.55 & $1.49,1.60$ & 1733 & 1.50 & $1.45,1.55$ \\
\hline T2 & 1465 & 1.46 & $1.41,1.51$ & 1744 & 1.52 & $1.47,1.57$ \\
\hline T3 & 1489 & 1.43 & $1.38,1.48$ & 1747 & 1.43 & $1.39,1.48$ \\
\hline $\begin{array}{c}P_{\text {trend }} \\
\text { AHEl-2010 }\end{array}$ & \multicolumn{3}{|c|}{0.004} & & \multicolumn{2}{|c|}{0.06} \\
\hline $\mathrm{T} 1$ & 1469 & 1.53 & $1.47,1.58$ & 1739 & 1.55 & $1.50,1.60$ \\
\hline T2 & 1466 & 1.45 & $1.40,1.50$ & 1729 & 1.47 & $1.43,1.52$ \\
\hline T3 & 1484 & 1.46 & $1.41,1.51$ & 1756 & 1.43 & $1.39,1.48$ \\
\hline $\begin{array}{l}P_{\text {trend }} \\
\text { aMED }\end{array}$ & \multicolumn{3}{|c|}{0.09} & & \multicolumn{2}{|c|}{0.002} \\
\hline $\mathrm{T} 1$ & 1539 & 1.53 & $1.47,1.59$ & 1865 & 1.50 & $1.45,1.56$ \\
\hline T2 & 1746 & 1.48 & $1.43,1.53$ & 2033 & 1.50 & $1.45,1.54$ \\
\hline T3 & 1134 & 1.40 & $1.34,1.47$ & 1326 & 1.44 & $1.38,1.50$ \\
\hline $\begin{array}{c}P_{\text {trend }} \\
\text { DASH }\end{array}$ & \multicolumn{3}{|c|}{0.007} & & \multicolumn{2}{|c|}{0.15} \\
\hline $\mathrm{T} 1$ & 1341 & 1.54 & $1.48,1.60$ & 1851 & 1.54 & $1.49,1.59$ \\
\hline T2 & 1448 & 1.48 & $1.43,1.54$ & 1757 & 1.46 & $1.41,1.51$ \\
\hline T3 & 1630 & 1.43 & $1.38,1.48$ & 1616 & 1.45 & $1.40,1.50$ \\
\hline$P_{\text {trend }}$ & \multicolumn{3}{|c|}{0.008} & & \multicolumn{2}{|c|}{0.02} \\
\hline
\end{tabular}

HEI, Healthy Eating Index; AHEI, Alternative Healthy Eating Index; aMED, alternate Mediterranean diet score; DASH, Dietary Approaches to Stop Hypertension.

* Values are geometric means $(95 \% \mathrm{Cl})$ of HOMA-IR across tertiles of dietary indices, adjusted for age, ethnicity, BMI, physical activity, smoking status, education and total energy intake. Participants with missing fasting insulin and glucose values were excluded for HOMA-IR analysis (remaining: men, 4419 and women, 5224).

significant in all subgroups. Only AHEI-2010 and DASH were significantly associated with higher HDL-cholesterol in women. A few other studies investigated the association of Mediterranean diet scores with adiponectin, and one intervention study and one out of two cross-sectional studies found positive relations ${ }^{(4)}$. A systematic review reported inverse associations between Mediterranean diet scores and CRP and other inflammation markers (three intervention studies and five out of seven cross-sectional studies), whereas three out of six cross-sectional studies found a weak inverse association between the HEI and $\mathrm{CRP}^{(4)}$.

Only the HEI-2010 and the DASH index take into account dairy products. As the DASH index focuses on low-fat dairy products, which had a stronger inverse association with T2D than total dairy intake ${ }^{(39)}$ and also a relation with adiponectin in previous studies ${ }^{(40)}$, this may explain that DASH was the only index positively associated with adiponectin. In addition, only the AHEI-2010 and DASH index take into account SSB consumption, which was related to higher TAG and lower HDLcholesterol $^{(41)}$. Higher AHEI scores were also related to higher adiponectin and lower $\mathrm{CRP}$ in the $\mathrm{NHS}^{(42)}$, whereas in the current study, a significant association of AHEI-2010 with CRP but not with adiponectin was detected. This may be possibly due to differences between the NHS and our cohort with regard to index versions, use of repeated $v$. one QFFQ, and ethnic composition, as adiponectin and other biomarker levels differed across ethnic groups in the MEC ${ }^{(43)}$.

Strengths of the current analysis include the prospective design, in particular, the availability of biomarkers and HOMAIR on an average of 10 years after dietary assessment at cohort entry as well as a large number of incident cases of T2D identified over many years with very strict diagnostic criteria: that is, self-reports and confirmation by administrative data. As for T2D status, we emphasised specificity in disease classification to avoid misclassification of healthy individuals as cases although a T2D diagnosis may have been missed for a small proportion of cohort members and for self-reported cases not confirmed by administrative data. The QFFQ was designed for the relevant ethnic groups who vary widely in dietary habits and was validated using $24-\mathrm{h}$ recalls ${ }^{(13)}$. The one-time dietary assessment by self-reported QFFQ was a limitation, which may have led to imprecise estimates of long-term dietary exposure. As a further limitation of this study, the relatively small size of the biomarker subcohort with an ethnic distribution unlike the full cohort, did not allow for a formal mediation analysis. The inclusion of 5 major ethnic groups residing in the United States who completed the same questionnaires and followed the same biomarker protocol is a unique contribution to the field because it allows direct comparison across ethnic groups. However, the results from ethnic groups in Hawaii and California may not be transportable to Hispanics, African and Asian Americans living elsewhere with different nutritional exposures and cultural conditions.

In conclusion, in this MEC, individuals with a higher compared with a lower score of three out of four dietary indices that quantify dietary recommendations based on scientific evidence had a lower incidence of T2D and all indices were related to lower HOMA-IR levels in at least one sex group. Although the strongest associations were detected for the DASH, higher scores for the AHEI-2010 and aMED also predicted a lower T2D risk. The relation of diet indices with biomarkers offers plausible biological mechanisms - that is, 

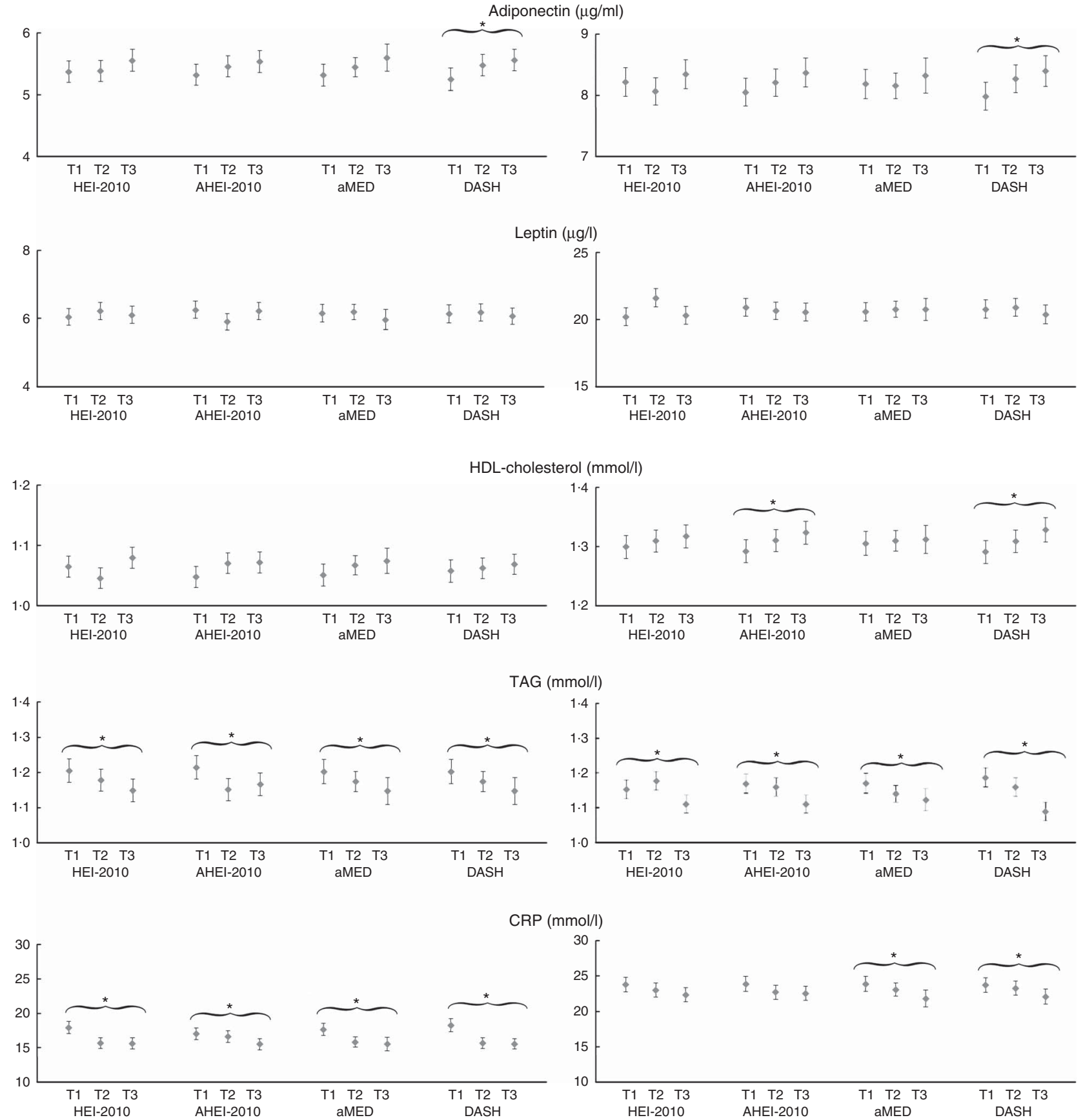

Fig. 2. Biomarkers across tertiles $(\mathrm{T})$ of Dietary Indices in the Multiethnic Cohort biomarker subcohort. Values are geometric means and $95 \% \mathrm{Cl}$ of biomarkers across tertiles of dietary indices adjusted for age, physical activity, smoking status, education, total energy intake, BMl and ethnicity, separately for men $(n 4661)$ and women ( $n$ 5399). Dietary index score range by tertile in men: Healthy Eating Index (HEI)-2010: T1: 30.3-61.2, T2: 61.2-70.9, T3: 70.9-95.9; Alternative Healthy Eating Index (AHEI)-2010: T1: 31.9-59.7, T2: 59.7-67.9, T3: 67.9-99.4; alternate Mediterranean diet score (aMED): T1: 0-3, T2: 4-5, T3: 6-9; Dietary Approaches to Stop Hypertension (DASH): T1: 11-21, T2: 22-25, T3: 26-38; and in women: HEl-2010: T1: 26.4-66.1, T2: 66.1-75.6, T3: 75.6-96.8; AHEI-2010: T1: 30.1-61.1, T2: 61.1-68.8, T3: 68.9-92.7; aMED: T1: 0-3, T2: 4-5, T3: 6-9; DASH: T1: 10-22, T2: 23-26, T3: 27-37. CRP, C-reactive protein. Because of space considerations, more decimal places are not presented, but with more decimal places, the limits do not overlap. ${ }^{*}$ Bracketed tertiles show statistically significant trends across tertiles $(P<0.05)$.

inflammation, adipokines and dyslipidaemia - by which diet quality may affect metabolic parameters involved in T2D aetiology. Future studies need to perform a formal mediation analysis to test whether the identified biomarkers indeed mediate the diet-T2D relation.

\section{Acknowledgements}

The Multiethnic Cohort was supported by NCI grant R37CA54281 (principal investigator (PI): L. N. K.) and U01CA164973 (PI: L. L./C. A. H./L. R. Wilkens). The recruitment 
of Native Hawaiians was funded by grant DAMD 17-94-T-4184 (PI: A. Nomura). The diabetes project was funded by R21DK073816 (PI: G. M.). S. J. was supported by a postdoctoral fellowship from the German Research Foundation (DFG, JA 2564/1-1).

L. L., C. A. H., L. N. K. designed research; A. A. F. provided essential analytic services and measured the biomarkers; C. J. B. developed nutritional support and developed the diet scores; S. J. and G. M. analysed the data and wrote the paper; S. J., C. J. B., A. A. F., L. L., Y. B. S., K. R. M., C. A. H., L. N. K., Y. B. S., G. M. contributed to the interpretation of the data, the critical revision of the article for important intellectual content and to the final approval of the version to be published. G. M. had primary responsibility for the final content.

The authors declare that there are no conflicts of interest.

\section{References}

1. Schwingshackl L \& Hoffmann G (2015) Diet quality as assessed by the Healthy Eating Index, the Alternate Healthy Eating Index, the Dietary Approaches to Stop Hypertension score, and health outcomes: a systematic review and meta-analysis of cohort studies. J Acad Nutr Diet 115, 780-800.

2. Schwingshackl L, Missbach B, Konig J, et al. (2015) Adherence to a Mediterranean diet and risk of diabetes: a systematic review and meta-analysis. Public Health Nutr 18, 1292-1299.

3. Hu FB (2002) Dietary pattern analysis: a new direction in nutritional epidemiology. Curr Opin Lipidol 13, 3-9.

4. Barbaresko J, Koch M, Schulze MB, et al. (2013) Dietary pattern analysis and biomarkers of low-grade inflammation: a systematic literature review. Nutr Rev 71, 511-527.

5. Sattar N, Wannamethee SG \& Forouhi NG (2008) Novel biochemical risk factors for type 2 diabetes: pathogenic insights or prediction possibilities? Diabetologia 51, 926-940.

6. Chen GC, Qin LQ \& Ye JK (2014) Leptin levels and risk of type 2 diabetes: gender-specific meta-analysis. Obes Rev 15, 134-142.

7. Lopez-Jaramillo P (2016) The role of adiponectin in cardiometabolic diseases: effects of nutritional interventions. J Nutr 146, 422S-426S.

8. Maskarinec G, Grandinetti A, Matsuura G, et al. (2009) Diabetes prevalence and body mass index differ by ethnicity: the Multiethnic Cohort. Ethn Dis 19, 49-55.

9. International Diabetes Federation (2015) IDF Diabetes Atlas. http://www diabetesatlas org/ (accessed July 2016).

10. Jacobs S, Harmon BE, Boushey CJ, et al. (2015) A prioridefined diet quality indexes and risk of type 2 diabetes: the Multiethnic Cohort. Diabetologia 58, 98-112.

11. Laubner K, Kieffer TJ, Lam NT, et al. (2005) Inhibition of preproinsulin gene expression by leptin induction of suppressor of cytokine signaling 3 in pancreatic beta-cells. Diabetes 54, 3410-3417.

12. Kolonel LN, Henderson BE, Hankin JH, et al. (2000) A multiethnic cohort in Hawaii and Los Angeles: baseline characteristics. Am J Epidemiol 151, 346-357.

13. Stram DO, Hankin JH, Wilkens LR, et al. (2000) Calibration of the dietary questionnaire for a multiethnic cohort in Hawaii and Los Angeles. Am J Epidemiol 151, 358-370.

14. Murphy SP (2002) Unique nutrition support for research at the Cancer Research Center of Hawaii. Hawaii Med J 61, 15, 17.

15. Harmon BE, Boushey CJ, Shvetsov YB, et al. (2015) Associations of key diet-quality indexes with mortality in the
Multiethnic Cohort: the Dietary Patterns Methods Project. Am J Clin Nutr 101, 587-597.

16. Guenther PM, Casavale KO, Reedy J, et al. (2013) Update of the Healthy Eating Index: HEI-2010. J Acad Nutr Diet 113, 569-580.

17. Guenther PM, Reedy J \& Krebs-Smith SM (2008) Development of the Healthy Eating Index-2005. J Am Diet Assoc 108, 1896-1901.

18. Chiuve SE, Fung TT, Rimm EB, et al. (2012) Alternative dietary indices both strongly predict risk of chronic disease. $J$ Nutr 142, 1009-1018.

19. Fung TT, Hu FB, Holmes MD, et al. (2005) Dietary patterns and the risk of postmenopausal breast cancer. Int J Cancer 116, 116-121.

20. Fung TT, McCullough ML, Newby PK, et al. (2005) Diet-quality scores and plasma concentrations of markers of inflammation and endothelial dysfunction. Am J Clin Nutr 82, 163-173.

21. Fung TT, Chiuve SE, McCullough ML, et al. (2008) Adherence to a DASH-style diet and risk of coronary heart disease and stroke in women. Arch Intern Med 168, 713-720.

22. Setiawan VW, Virnig BA, Porcel J, et al. (2015) Linking data from the Multiethnic Cohort Study to Medicare data: linkage results and application to chronic disease research. Am J Epidemiol 181, 917-919.

23. Maskarinec G, Erber E, Grandinetti A, et al. (2009) Diabetes incidence based on linkages with health plans: the multiethnic cohort. Diabetes 58, 1732-1738.

24. State of California Office of Statewide Health Planning \& Development (2015) Health Care Information Division Patient Discharge Data. http://www oshpd ca gov/HID/ Products/PatDischargeData/PublicDataSet/index html (accessed September 2015).

25. Manson JE, Rimm EB, Stampfer MJ, et al. (1991) Physical activity and incidence of non-insulin-dependent diabetes mellitus in women. Lancet 338, 774-778.

26. Choi HK, Willett WC, Stampfer MJ, et al. (2005) Dairy consumption and risk of type 2 diabetes mellitus in men: a prospective study. Arch Intern Med 165, 997-1003.

27. Margolis KL, Lihong Q, Brzyski R, et al. (2008) Validity of diabetes self-reports in the Women's Health Initiative: comparison with medication inventories and fasting glucose measurements. Clin Trials 5, 240-247.

28. Okura Y, Urban LH, Mahoney DW, et al. (2004) Agreement between self-report questionnaires and medical record data was substantial for diabetes, hypertension, myocardial infarction and stroke but not for heart failure. J Clin Epidemiol 57, 1096-1103.

29. Jimenez-Pavon D, Sese MA, Huybrechts I, et al. (2013) Dietary and lifestyle quality indices with/without physical activity and markers of insulin resistance in European adolescents: the HELENA study. Br J Nutr 110, 1919-1925.

30. Shirani F, Salehi-Abargouei A \& Azadbakht L (2013) Effects of Dietary Approaches to Stop Hypertension (DASH) diet on some risk for developing type 2 diabetes: a systematic review and meta-analysis on controlled clinical trials. Nutrition 29, 939-947.

31. Cespedes EM, Hu FB, Tinker L, et al. (2016) Multiple Healthful Dietary Patterns and Type 2 Diabetes in the Women's Health Initiative. Am J Epidemiol 183, 622-633.

32. Liese AD, Nichols M, Sun X, et al. (2009) Adherence to the DASH Diet is inversely associated with incidence of type 2 diabetes: the insulin resistance atherosclerosis study. Diabetes Care 32, 1434-1436.

33. InterAct Consortium (2014) Adherence to predefined dietary patterns and incident type 2 diabetes in European populations: EPIC-InterAct Study. Diabetologia 57, 321-333.

34. Otto MC, Padhye NS, Bertoni AG, et al. (2015) Everything in Moderation-Dietary Diversity and Quality, Central Obesity and Risk of Diabetes. PLOS ONE 10, e0141341. 
35. Fung TT, McCullough M, van Dam RM, et al. (2007) A prospective study of overall diet quality and risk of type 2 diabetes in women. Diabetes Care 30, 1753-1757.

36. de Koning L, Chiuve SE, Fung TT, et al. (2011) Diet-quality scores and the risk of type 2 diabetes in men. Diabetes Care $\mathbf{3 4}$, $1150-1156$.

37. Kodama K, Tojjar D, Yamada S, et al. (2013) Ethnic differences in the relationship between insulin sensitivity and insulin response: a systematic review and meta-analysis. Diabetes Care 36, 1789-1796.

38. Sharma S, Sheehy T \& Kolonel L (2014) Sources of vegetables, fruits and vitamins $\mathrm{A}, \mathrm{C}$ and $\mathrm{E}$ among five ethnic groups: results from a multiethnic cohort study. Eur J Clin Nutr 68, 384-391.

39. Gao D, Ning N, Wang C, et al. (2013) Dairy products consumption and risk of type 2 diabetes: systematic review and dose-response meta-analysis. PLOS ONE 8, e73965.
40. Niu K, Kobayashi Y, Guan L, et al. (2013) Low-fat dairy, but not whole-/high-fat dairy, consumption is related with higher serum adiponectin levels in apparently healthy adults. Eur J Nutr 52, 771-778.

41. de Koning L, Malik VS, Kellogg MD, et al. (2012) Sweetened beverage consumption, incident coronary heart disease, and biomarkers of risk in men. Circulation $\mathbf{1 2 5}$, 1735-1741 S1.

42. Fargnoli JL, Fung TT, Olenczuk DM, et al. (2008) Adherence to healthy eating patterns is associated with higher circulating total and high-molecular-weight adiponectin and lower resistin concentrations in women from the Nurses' Health Study. Am J Clin Nutr 88, 1213-1224.

43. Morimoto Y, Conroy SM, Ollberding NJ, et al. (2014) Ethnic differences in serum adipokine and C-reactive protein levels: the multiethnic cohort. Int $J$ Obes (Lond) 38, $1416-1422$. 